\title{
Ultralong Raman Fiber Lasers as Virtually Lossless Optical Media
}

\author{
Juan Diego Ania-Castañón, Tim J. Ellingham, R. Ibbotson, X. Chen, L. Zhang, and Sergei K. Turitsyn \\ Photonics Research Group, School of Engineering and Applied Science, Aston University, Birmingham, B47ET, United Kingdom
}

(Received 19 September 2005; published 18 January 2006)

\begin{abstract}
By transforming the optical fiber span into an ultralong cavity laser, we experimentally demonstrate quasilossless transmission over long (up to $75 \mathrm{~km}$ ) distances and virtually zero signal power variation over shorter (up to $20 \mathrm{~km}$ ) spans, opening the way for the practical implementation of integrable nonlinear systems in optical fiber. As a by-product of our technique, the longest ever laser (to the best of our knowledge) has been implemented, with a cavity length of $75 \mathrm{~km}$. A simple theory of the lossless fiber span, in excellent agreement with the observed results, is presented.
\end{abstract}

DOI: 10.1103/PhysRevLett.96.023902

PACS numbers: 42.81.Dp, 42.55.Ye, 42.65.Dr

Introduction. - Research on fiber optic technologies has expanded dramatically over the past several decades. Optical fiber underpins the material revolution in modern photonics with numerous applications from telecommunications and lasers to medicine and sensing. In long distance communication fiber links an optical signal can potentially propagate hundreds of $\mathrm{km}$ before reamplification, and even more excitingly, it is possible to use the intrinsic material properties of silica fibres to achieve distributed amplification along the fiber at the signal wavelengths by providing external optical pumping [1]. This way, fiber itself can be exploited as an amplifying medium, combating the attenuation of the transmitted signal within the fiber span. In recent years, different techniques have been proposed to achieve an even distribution of the gain along the fiber span (aiming at ideally lossless transmission), such as bidirectional pumping schemes, effective fiber core area management, and the use of higher-order pumping structures [24]. Lossless transmission, a long-term dream goal of optical communications, would imply a minimization of the amplified spontaneous emission (ASE) noise production, effectively offering the best possible trade-off between accumulated nonlinearities and ASE buildup [5]; thus, it would guarantee the best possible performance from any system in which ASE is the only meaningful source of noise. Indeed, a transmission system with no effective spatial attenuation would be ideal both for low signal power applications and for systems/devices in which high nonlinearities play a positive role (e.g., soliton based) [69]. It would also lead to a new interesting connection between fiber optics and the theory of nonlinear integrable systems. It is known that the propagation of an optical signal along a cascaded fiber link with periodic amplification is governed under certain conditions by a model close to the nonlinear Schrödinger equation (NLSE) [6,9]. The NLSE is an example of an integrable nonlinear model (here, integrability refers to the existence of an infinite number of conserved integrals of motion), displaying dynamical properties different from those of general (non- integrable) nonlinear systems. The powerful inverse scattering transform method (conceptually, a nonlinear analogous to the Fourier transform) can be applied to integrable nonlinear systems, opening fundamentally new possibilities for their theoretical analysis [10]. However, the inevitable presence of losses has prevented the NLSE from ever being applied to optical fiber in its true integrable version, even though it has been used in some situations to describe evolution of the averaged optical field [6,9]. In this Letter, we demonstrate the first experimental implementation of an integrable nonlinear system in a fiber waveguide where power losses vs distance are continuously compensated with ultrahigh precision by propagating the signal inside an ultralong laser, effectively creating a lossless fiber span (with amplitude variations below the accuracy of our measurement techniques). A relatively long link of up to $25 \mathrm{~km}$ with ultralow power excursion could be used in data processing applications, opening the way for the development of a practical framework for the design of photonic systems/devices based on the mathematical concept of integrable nonlinear systems, with functionalities beyond those of their linear counterparts. As a by-product, we have experimentally implemented what we believe is the longest ever laser, with a $75 \mathrm{~km}$ cavity length.

Design and experimental setup.-The design of the quasilossless span, recently proposed by one of the authors [11], is schematically depicted in Fig. 1. It includes two equal-power, depolarized primary pumps launched from both edges of an elementary cell comprised of any optical fiber [in our experiment, silica-based standard monomode fiber (SMF)]. The bidirectional pumping structure is combined with two fiber-Bragg-grating (FBG) reflectors positioned at both ends of the cell. In our experiment, the depolarized primary pumps were operated at a wavelength $\lambda_{1}=c / \nu_{1}$ of about $1365 \mathrm{~nm}$. The central frequency of the gratings $\left(\nu_{2}\right)$ is set to be in the vicinity of the primary pumps' first Stokes (here $\lambda_{2}=c / \nu_{2}=1455 \mathrm{~nm}$ ), so that the pair of gratings and the fiber span create a cavity for the radiation at this frequency. If the power in the primary 


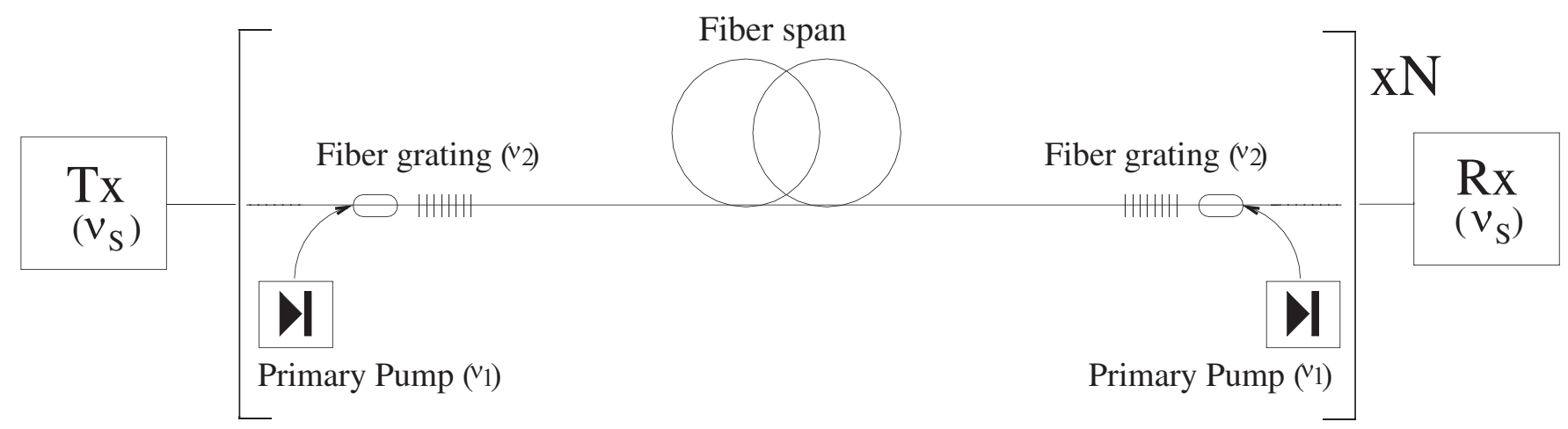

FIG. 1. Schematic depiction of the system.

pumps is above the threshold necessary for stimulated Raman scattering to overcome fiber attenuation at the central frequency of the reflectors, the whole span becomes an ultralong laser and a stable secondary pump at such frequency is generated in the cavity from the ASE. Given the symmetry of the primary pumps, the combined forward- and backward-propagating power of the secondary pump presents very little variation vs distance along the span, and can therefore be used to provide a nearly constant Raman gain at the signal frequency (in our experiment, corresponding to a wavelength of $1550 \mathrm{~nm}$, which is typical for optical fiber transmission applications). Moreover, the power level of the secondary pump can be easily adjusted by controlling the power in the primary pumps, so that the signal gain provided closely matches fiber attenuation at every step of the propagation. The same structure can be successfully applied to long and short fiber spans. We have measured signal variations using optical time-domain reflectometry, employing a low power $(<0.1 \mathrm{~mW})$ signal to monitor power excursion at
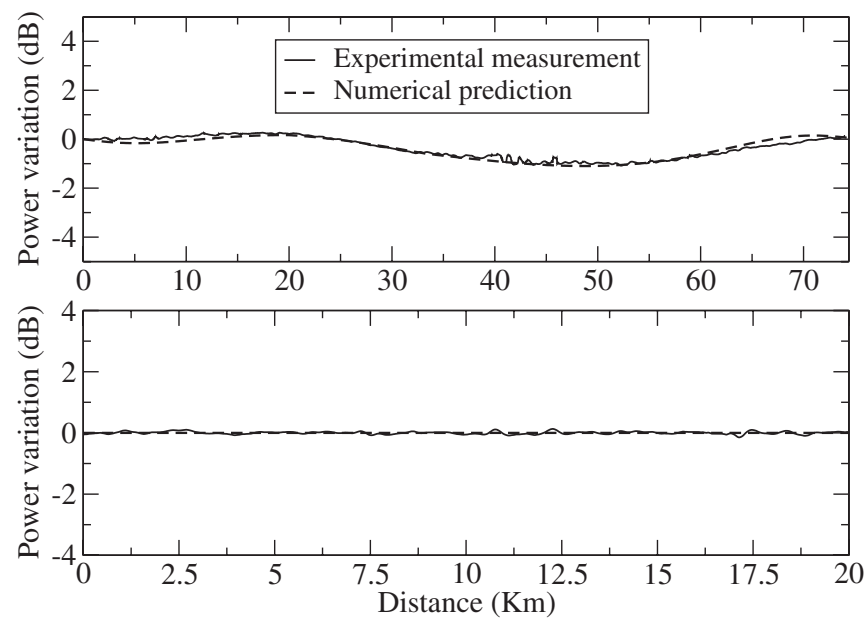

FIG. 2. Signal power excursion for two different transmission lengths. The solid lines correspond to the experimental results, whereas the dashed line shows the theoretical prediction.
$1550 \mathrm{~nm}$. Figure 2 depicts signal power evolution in the fiber span for two different transmission lengths, both as measured and as predicted by the theoretical model described below. The top figure corresponds to a $75 \mathrm{~km}$ span, in which quasilossless transmission was achieved by pumping the cavity with $1322 \mathrm{~mW}$ from the primary pump lasers. The reflectivity of the FBGs, centered at $1455 \mathrm{~nm}$, was about 0.98. Because of minor wavelength differences between the two primary lasers (emitting at 1364.2 and $1365.4 \mathrm{~nm}$, respectively) the pump powers of the forward and backward pump had to be slightly unbalanced. Even with this artificial limitation, the measured signal power variation over $75 \mathrm{~km}$ standard SMF was $1.35 \mathrm{~dB}$, only $0.1 \mathrm{~dB}$ above the $1.25 \mathrm{~dB}$ predicted by the theoretical model. The bottom case corresponds to a $20 \mathrm{~km}$ fiber span. The pump power used in this case was $351 \mathrm{~mW}$, and the measured variation is below the precision of our experimental setup, with the theory predicting a variation smaller than $0.001 \mathrm{~dB}$, or virtually lossless transmission. All measured pump powers were also in excellent agreement with the theoretical predictions. As for the cavitygenerated secondary pumps, simulations show that the reflected power into the span at the fiber-Bragg-grating reflectors is about $38 \mathrm{~mW}$ for the $75 \mathrm{~km}$ link and $51 \mathrm{~mW}$ for the $20 \mathrm{~km}$ link (the combined forward and backward power at the reflectors is, respectively, 77 and $103 \mathrm{~mW}$ ). The measured noise performance was excellent, with an equivalent ASE noise figure of $-4 \mathrm{~dB}$ for the $75 \mathrm{~km}$ lossless span, whereas simulations suggest that in-band double Rayleigh backscattering noise power is more than 1 order of magnitude lower than ASE, and more than $55 \mathrm{~dB}$ lower than signal power at the end of the span. A wavelengthtunable signal source was used for monitoring total gain and power excursion in the $1520-1580 \mathrm{~nm}$ region, showing that our setup can provide quasilossless transmission over a broad bandwidth. In particular, total output signal power variation in the $1530-1566 \mathrm{~nm}$ region was below $1.5 \mathrm{~dB}$, with power excursions not higher than $2.5 \mathrm{~dB}$ for any wavelength in the range. No stability problems were observed in continuous operation for neither the short nor the long link, and numerical simulations show it as being 
stable against cavity length, signal power, and temperature variations. An additional by-product of the extreme cavity lengths used is an ultradense packing of the fiber laser cavity modes. This negates the possibility of beating noise being present in the secondary pump due to mode sweeping. The total fiber attenuation (i.e., the total power excursion if amplification would have taken place at the end of the span) was $15 \mathrm{~dB}$ for the $75 \mathrm{~km}$ span and $4 \mathrm{~dB}$ for the $20 \mathrm{~km}$ link.

Quasilossless span theory.-In our theoretical analysis we employ a twofold approach. First, we numerically study the full model for the evolution of the average power of signal and pumps, taking into account pump depletion, as well as ASE and double Rayleigh backscattering as sources of noise [11]. On the other hand, it is useful to simplify the model to gain insight into the basic physical effects and find analytical estimates for the required pump parameters. Neglecting noise terms, the equations describing the mainorder effects in the proposed scheme are:

$\frac{d P_{1}^{ \pm}}{d z}=\mp \alpha_{1} P_{1}^{ \pm} \mp g_{1} \frac{\nu_{1}}{\nu_{2}}\left(P_{2}^{+}+P_{2}^{-}\right) P_{1}^{ \pm}$,

$\frac{d P_{2}^{ \pm}}{d z}=\mp \alpha_{2} P_{2}^{ \pm} \mp \frac{g_{2} \nu_{2}}{\nu_{S}} P_{S} P_{2}^{ \pm} \pm g_{1}\left(P_{1}^{+}+P_{1}^{-}\right) P_{2}^{ \pm}$,

$\frac{d P_{S}}{d z}=-\alpha_{S} P_{S}+g_{2}\left(P_{2}^{+}+P_{2}^{-}\right) P_{S}$

where $P_{1}^{ \pm}$and $P_{2}^{ \pm}$represent the forward (+) and backward $(-)$ propagating average powers for the initial (index 1) and the Stokes-generated secondary (2) pumping waves, at frequencies $\nu_{1}$ and $\nu_{2}$, respectively. The secondary pumps generated in the ultralong cavity laser provide direct amplification to the signal (with the power $P_{S}$ ) at frequency $\nu_{S}$. Here $g_{1}$ represents the Raman gain coefficient for the transition from primary pump to secondary pump and $g_{2}$ the coefficient for the transition from secondary pump to

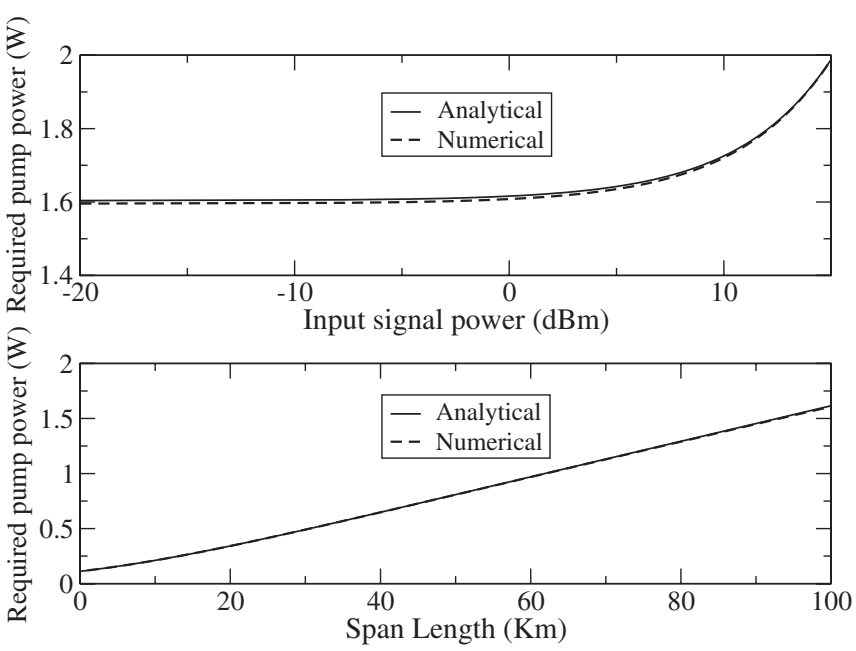

FIG. 3. Top: Total required pump power in the primary pumps vs input signal power. Solid line-numerics; dashed lineanalytics. Bottom: Idem vs span length for a $1 \mathrm{~mW}(0 \mathrm{dBm})$ signal.

signal; $\alpha_{j}$ stand for the fiber attenuation coefficients at the corresponding wavelengths defined by the index $j$. Primary pump power is adjusted to recover initial signal power at $z=L$, imposing constraint $\int_{0}^{L}\left(P_{2}^{+}+P_{2}^{-}\right) d z=\alpha_{S} L / g_{2}$. In addition, the following boundary conditions apply: $P_{2}^{+}(0)=R_{1} P_{2}^{-}(0) ; \quad P_{2}^{-}(L)=R_{2} P_{2}^{+}(L) ; \quad P_{1}^{+}(0)=P_{P}^{+}$; $P_{1}^{-}(L)=P_{P}^{-} ; P_{S}(0)=P_{\text {in }}$, where the $R_{i}$ are the reflectivities of the fiber-Bragg gratings, the $P_{P}^{ \pm}$represents the corresponding input primary pump powers, and $P_{\text {in }}$ is the input signal power. For the sake of generality, reflectivity for each grating and input powers for each primary pump are assumed to be different (in our experiment they are chosen to be as close to each other as possible to preserve the symmetry of the setup). After straightfoward manipulations, the following relation is derived:

$$
\begin{aligned}
P_{2}^{+}(0)\left[\frac{1-R_{2}}{\sqrt{R_{1} R_{2}}}+\frac{1-R_{1}}{R_{1}}\right]= & \left(P_{P}^{+}+P_{P}^{-}\right)(1-\epsilon) \frac{\nu_{2}}{\nu_{1}}-\frac{\alpha_{2} \alpha_{S} L}{g_{2}}-\frac{\nu_{2} \alpha_{1} \alpha_{2} L}{\nu_{1} g_{1}}+\frac{\nu_{2} \alpha_{1}}{2 \nu_{1} g_{1}} \ln \left(R_{1} R_{2}\right) \\
& -\left(\alpha_{S}+\alpha_{1} \frac{\nu_{2}^{2} g_{2}}{\nu_{S} \nu_{1} g_{1}}\right) \int_{0}^{L} P_{S} d z,
\end{aligned}
$$

where $\epsilon=\exp \left(-\alpha_{1} L-\frac{\nu_{1} g_{1}}{\nu_{2} g_{2}} \alpha_{S} L\right)$.

From this relation it can be seen that if the we assume for simplicity that $R_{i}$ are exactly equal to unity (typically $R_{i} \sim$ 0.97 to 0.99 ), and that signal power varies only slightly along the line once the secondary pump is stable, then the required power in the primary pumps can be estimated as

$$
\begin{aligned}
\left(P_{P}^{+}+P_{P}^{-}\right) \approx & \left(\frac{\alpha_{S} \nu_{1}}{g_{2} \nu_{2}}+\frac{\alpha_{1}}{g_{1}}\right) \frac{\alpha_{2} L}{1-\epsilon} \\
& +\frac{\nu_{1}}{\nu_{2}(1-\epsilon)}\left(\alpha_{S}+\alpha_{1} \frac{\nu_{2}^{2} g_{2}}{\nu_{S} \nu_{1} g_{1}}\right) P_{\text {in }} L
\end{aligned}
$$

Figure 3 compares the analytical and the numerical predictions for the required input pump power, with a fiber link comprised of standard single-mode fiber. The agreement is excellent in all cases, whether we are plotting the dependence with input signal power for a fixed fiber link length (100 km in Fig. 3) or the variation vs length of the optical link for a fixed input signal power $(1 \mathrm{~mW})$.

Next we have to check that the lasing condition for the secondary pump, $\left(\int_{0}^{L}\left[-\alpha_{2}+g_{1}\left(P_{1}^{+}+P_{1}^{-}\right)-\right.\right.$ $\left.\left.g_{2} \frac{\nu_{2}}{\nu_{S}} P_{S}\right] d z>0\right)$, is satisfied. In other terms, this is the condition providing growth of a radiation seeded at the 


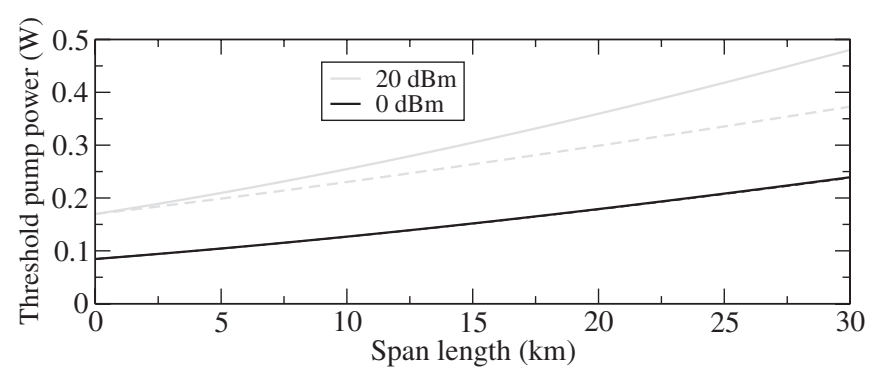

FIG. 4. Lower (dashed) and upper (solid) limits for the lasing threshold vs length of the fiber link for a $1 \mathrm{~mW}$ (black) and a $100 \mathrm{~mW}$ (gray) input signal

secondary pump frequency $\nu_{2}$. By neglecting the Ramaninduced depletion of the primary pumps (initial secondary pump power is at ASE level), and choosing adequate upper bound (no signal attenuation in the fiber) and lower bound (no signal gain in the fiber) for the integrated signal power, the threshold pump power $P_{\text {th }}$ that guarantees lasing at a frequency $\nu_{2}$ can be estimated as

$$
\begin{aligned}
& \frac{1}{2}\left[\frac{\alpha_{1} \alpha_{2} L}{g_{1}\left(1-e^{-\alpha_{1} L}\right)}+\frac{g_{2} \alpha_{1}\left(1-e^{-\alpha_{S} L}\right) \nu_{2}}{g_{1} \alpha_{S}\left(1-e^{-\alpha_{1} L}\right) \nu_{S}} P_{\text {in }}\right]<P_{\text {th }}<\frac{L}{2} \\
& \quad \times\left[\frac{\alpha_{1} \alpha_{2}}{g_{1}\left(1-e^{-\alpha_{1} L}\right)}+\frac{g_{2} \alpha_{1} \nu_{2}}{g_{1}\left(1-e^{-\alpha_{1} L}\right) \nu_{S}} P_{\text {in }}\right] .
\end{aligned}
$$

Both limits are very close to each other in most cases, and virtually indistinguishable (see Fig. 4) for an input signal power of $1 \mathrm{~mW}(0 \mathrm{dBm})$, but their separation increases for long spans with high signal powers, as the effect of depletion becomes significant, as observable in the example for a very high $100 \mathrm{~mW}$ signal. Note that a minimum pump power is required for lasing, independently of the length of the fiber link. Of course, the required power for quasilossless transmission, shown in Fig. 3 is always higher than the lasing threshold, and as such, it presents a similar hard threshold, no matter how short the link.

Conclusion. -We have experimentally implemented an effectively lossless fiber span by propagating a signal through an ultralong Raman laser pumped at a frequency two Raman Stokes above that of the signal, and created in the process a laser cavity of $75 \mathrm{~km}$, the longest ever to the extent of our knowledge. We have demonstrated the ability of the scheme to provide quasilossless transmission over long (up to $75 \mathrm{~km}$ ) fiber spans, as well as to achieve almost zero optical signal variation over shorter distances $(20 \mathrm{~km}$ or less). Such lossless fiber links could be key elements for the practical implementation of integrable nonlinear systems in optical fiber devices. We have also provided a theoretical analysis of the quasilossless system that shows excellent agreement with the experiments.

[1] R. H. Stolen, and E. P. Ippen, Appl. Phys. Lett. 22, 276 (1973).

[2] T. Okuno, T. Tsuzaki, and M. Nishimura, IEEE Photonics Technol. Lett. 13, 806 (2001).

[3] J.-C. Bouteiller, K. Brar, and C. Headley, in Proceedings of the European Conference on Optical Communications 2002, Copenhagen, Denmark (Opt. Soc. Am., Washington, DC, 2002), p. S3.04.

[4] K. Rottwitt, A. Stentz, T. Nielson, P. Hansen, K. Feder, and $\mathrm{K}$. Walker, in Proceedings of the European Conference on Optical Communications 99, Nice, France (Opt. Soc. Am., Washington, DC, 1999), p. II.

[5] V.E. Perlin, and H. G. Winful, in Proceedings of the Optical Fiber Conference 2002, Anaheim, California (Opt. Soc. Am., Washington, DC, 2002), paper WB1, p. 178.

[6] A. Hasegawa and Y. Kodama, Solitons in Optical Communications (Clarendon, Oxford, NY, 1995).

[7] L.F. Mollenauer, R. H. Stolen, and J.P. Gordon, Phys. Rev. Lett. 45, 1095 (1980).

[8] Yu. S. Kivshar and G. P. Agrawal, Optical Solitons: From Fibers to Photonic Crystals (Academic, San Diego, 2003).

[9] E. Iannone, F. Matera, A. Mecozzi, and M. Settembre, Nonlinear Optical Communication Networks (John Wiley \& Sons, New York, 1998).

[10] V.E. Zakharov, A. B. Shabat, Sov. Phys. JETP 34, 62 (1972).

[11] J. D. Ania-Castañón, Opt. Express 12, 4372 (2004). 